\title{
HOPF-TYPE RIGIDITY FOR NEWTON EQUATIONS
}

\author{
Misha Bialy and Leonid Polterovich
}

\section{Introduction and main results}

Let $\mathbb{T}^{n}$ be a Euclidean torus. The motion of the classical particle on $\mathbb{T}^{n}$ in the presence of a time-dependent potential $U: \mathbb{T}^{n} \times \mathbb{R} \rightarrow \mathbb{R}$ is described by the Newton equation

$$
\nabla_{\dot{q}} \dot{q}=-\nabla U(q, t)
$$

which is the Euler-Lagrange equation for the variational problem

$$
\int \frac{1}{2}|\dot{q}|^{2}-U(q, t) d t \rightarrow \operatorname{extr}
$$

In what follows we assume the potential $U$ to be $T$-periodic in time. In the present note we prove the following Hopf-type rigidity result:

Theorem 1.B. The Newton equation (1.A) has no conjugate points if and only if the potential $U$ does not depend on $q$, that is, $\nabla U(q, t) \equiv 0$.

We refer the reader to $[\mathrm{ATF}]$ for the standard definition of conjugate points. The proof of $1 . \mathrm{B}$ is given in $\S 2$.

In the case when the potential $U$ is time-independent a stronger statement was proved by A. Knauf [K]. Namely, if a potential system has no conjugate points in a sufficiently high energy level then $U$ is constant. Knauf uses a Hopf's method (see $[\mathrm{H}],[\mathrm{G}]$ ), which relies heavily on the integration of certain curvature-type quantities over the compact energy level. In the time-dependent situation the energy is no longer conserved, and hence the phase space is essentially non-compact. This forms the main difficulty of our problem, and some new tools are needed.

Received September 20, 1995.

First author supported by German-Israeli Foundation grant G-0275-025.06/93 
Remark 1.C. Our result remains true in a more general Riemannian context. Namely, the Newton equation (1.A) associated with an arbitrary Riemannian metric on the torus has no conjugate points if and only if the metric is flat and the potential does not depend on $q$. Indeed, the transformation $q(t) \mapsto q(\varepsilon t)$ maps the solutions of the Newton equation with potential $U(q, t)$ to the solutions of the Newton equation with potential $\varepsilon^{2} U(q, \varepsilon t)$. In particular, if the former system has no conjugate points then neither does the latter. In view of this, the Newton equation can be considered as a small perturbation of the equation of geodesics. This immediately implies that the Riemannian metric has no conjugate points, and must then be flat due to the Burago-Ivanov solution of the Hopf conjecture ([B-I]; see $[\mathrm{Ba}],[\mathrm{C}-\mathrm{K}]$ for the history and related discussions). The desired assertion follows now from 1.B.

The Hamiltonian counterpart of the Newton equation (1.A) is a flow $\varphi_{t}$ on $T^{*} \mathbb{T}^{n}$ generated by a Hamiltonian function $H(p, q, t)=\frac{1}{2}|p|^{2}+U(q, t)$. Let $\varphi=\varphi_{T}$ be the time- $T$-map of the flow. As a consequence of 1 .B we get the following:

Theorem 1.D. Suppose that the phase space $T^{*} \mathbb{T}^{n}$ is $C^{1}$-foliated by $\varphi$ invariant Lagrangian tori homologous to the zero section. Then the potential $U$ does not depend on $q$.

Proof. It is well known that every leaf $L$ of the invariant foliation carries a $\varphi$-invariant absolutely continuous measure. (Warning: such a measure does not necessarily exist if an invariant torus is not a leaf of an invariant foliation!) Then the natural projection to $\mathbb{T}^{n}$ of every trajectory lying on $L$ is a minimal extremal of the action functional (see $[\mathrm{B}-\mathrm{P}], 1.4$ ), and hence the system has no conjugate points. The needed assertion follows from 1.B.

\section{Hopf's method and Gibbs measure}

In this section we prove 1.B. Suppose that (1.A) has no conjugate points. Consider the corresponding extended Hamiltonian flow in the phase space $M=T^{*} \mathbb{T}^{n} \times S^{1}$ where $S^{1}=\mathbb{R} / T \mathbb{Z}$. Let $\mathcal{L}$ be the Lie derivative operator along this flow. As in the classical Hopf method (see $[\mathrm{H}],[\mathrm{G}]$ ) one can construct a measurable family $A(p, q, t)$ of symmetric $n \times n$ matrices on $M$ which is smooth along trajectories of the flow and satisfies the Riccati equation $\mathcal{L} A+A^{2}+\operatorname{Hess}_{q} U=0$. Moreover, $A$ and $\mathcal{L} A$ are uniformly bounded. 
Set $a(p, q, t)=\operatorname{tr} A(p, q, t)$. Then the inequality $\operatorname{tr} A^{2} \geq \frac{1}{n}(\operatorname{tr} A)^{2}$ implies that

$$
\mathcal{L} a+\frac{1}{n} a^{2}+\Delta_{q} U \leq 0
$$

It would be easy to complete the proof to the theorem if, following Hopf, we could integrate (2.A) over $M$. In order to handle non-compactness, we introduce a fastly decaying Gibbs measure

$$
d \mu_{U}=\exp \left\{-\frac{|p|^{2}}{2}-U(q, t)\right\} d p_{1} d q_{1} \cdot \ldots \cdot d p_{n} d q_{n} d t
$$

Let $\mathcal{E}_{U}$ be the space of all measurable uniformly bounded functions $a: M \rightarrow$ $\mathbb{R}$ which are smooth along the trajectories of the flow and whose Lie derivative $\mathcal{L} a$ is uniformly bounded. The following result is crucial for our purposes:

Lemma 2.B. Assume that the potential $U$ satisfies

$$
\int_{M}\left[\left(\frac{\partial U}{\partial t}\right)^{2}-\frac{4}{n}|\nabla U|^{2}\right] d \mu_{U}<0 \text {. }
$$

Then

$$
\int_{M}\left(\mathcal{L} a+\frac{1}{n} a^{2}+\Delta_{q} U\right) d \mu_{U}>0
$$

holds for every function $a \in \mathcal{E}_{U}$.

Proof. Integrating by parts and using the fact that the Liouville measure is invariant under the flow we get that

$$
\int_{M} \mathcal{L} a d \mu_{U}=\int_{M} a \frac{\partial U}{\partial t} d \mu_{U}
$$

Hence, the Cauchy-Schwartz inequality in $L^{2}\left(M, d \mu_{U}\right)$ implies that

$$
\int_{M} \mathcal{L} a d \mu_{U} \geq-\left(\int_{M} a^{2} d \mu_{U}\right)^{\frac{1}{2}}\left(\int_{M}\left(\frac{\partial U}{\partial t}\right)^{2} d \mu_{U}\right)^{\frac{1}{2}} .
$$


Also,

$$
\int_{M} \Delta_{q} U d \mu_{U}=\int_{M}|\nabla U|^{2} d \mu_{U} .
$$

Set $x=\left(\int_{M} a^{2} d \mu_{U}\right)^{\frac{1}{2}}$. Then we get that

$\int_{M}\left(\mathcal{L} a+\frac{1}{n} a^{2}+\Delta_{q} U\right) d \mu_{U} \geq \frac{1}{n} x^{2}-\left(\int_{M}\left(\frac{\partial U}{\partial t}\right)^{2} d \mu_{U}\right)^{\frac{1}{2}} \cdot x+\int_{M}|\nabla U|^{2} d \mu_{U}$.

Notice now that the left-hand side of (2.C) is just the discriminant of this quadratic function. The needed assertion follows immediately.

The next step is to apply the renormalization procedure of 1.C to our potential system. This procedure does not change the essential behavior of the system and, in a sense, allows one to work in a neighbourhood of the Euclidean geodesic flow. For $\varepsilon>0$ define a renormalized potential $U_{\varepsilon}(q, t)=\varepsilon^{2} U(q, \varepsilon t)$. Notice that $U_{\varepsilon}$ is periodic with the period $T_{\varepsilon}=T / \varepsilon$. Set $M_{\varepsilon}=T^{*} \mathbb{T}^{n} \times\left(\mathbb{R} / T_{\varepsilon} \mathbb{Z}\right)$.

Lemma 2.D. Assume that the potential $U$ depends non-trivially on $q$. Then the renormalized potential $U_{\varepsilon}$ satisfies the inequality (2.C), that is

$$
\int_{M_{\varepsilon}}\left[\left(\frac{\partial U_{\varepsilon}}{\partial t}\right)^{2}-\frac{4}{n}\left|\nabla U_{\varepsilon}\right|^{2}\right] d \mu_{U_{\varepsilon}}<0,
$$

provided $\varepsilon$ is small enough.

Proof. A straightforward computation shows that

$$
\int_{M_{\varepsilon}}\left(\frac{\partial U_{\varepsilon}}{\partial t}\right)^{2} d \mu_{U \varepsilon} \leq c_{1} \varepsilon^{5}
$$

while

$$
\int_{M_{\varepsilon}}\left|\nabla U_{\varepsilon}\right|^{2} d \mu_{U \varepsilon} \geq c_{2} \varepsilon^{3}
$$

where $c_{1}$ and $c_{2}$ are positive constants. The required assertion follows immediately. 
Now we are ready to finish off the proof of 1 .B. Let $\frac{|p|^{2}}{2}+U(q, t)$ be a potential system without conjugate points. Suppose that the potential $U$ depends non-trivially on $q$. Then after the renormalization (see 2.D) we can assume that the potential $U$ satisfies the inequality (2.C). Hence the function $a$ violates the inequality (2.A) in view of Lemma 2.B which is a contradiction. This completes the proof.

\section{Discussion}

3.A. Compactly supported potentials. Our method can be applied for other boundary conditions. Consider for instance the Newton equation for a potential $U: \mathbb{R}^{n} \times \mathbb{R} \rightarrow \mathbb{R}$ with compact support and a Riemannian metric on $\mathbb{R}^{n}$ which is flat outside a compact subset of $\mathbb{R}^{n}$. Using similar arguments as for the proof of $1 . \mathrm{B}, \mathrm{C}$ one can show that the Newton equation in this case has no conjugate points if and only if $g$ is flat and $U$ vanishes identically. Note that the flatness of the metric here, follows from a result by C. Croke who proved the E. Hopf theorem for the "compactly supported" case (see $[\mathrm{C}])$.

3.B. Which classes of variational problems enjoy the Hopf-type rigidity? This problem is still far from being understood. Besides Riemannian metrics and time-periodic potential systems, the Hopf-type rigidity holds for convex plane billiards. Namely, the first author proved (see [B]) that the only billiard without conjugate points is a circle. In [D] the Hopf-type rigidity is discussed in a two-dimensional Finsler framework. No other results in this direction are known to us.

3.C. Optical systems without conjugate points. Recall that a Hamiltonian system on $T^{*} \mathbb{T}^{n}$ is optical if it is generated by a fiber-wise strictly convex Hamiltonian function. Given an optical system without conjugate points, does it admit a smooth invariant foliation by Lagrangian tori?

This question was posed in $[\mathrm{C}-\mathrm{K}]$ for the Finsler case. However, it makes sense in the general setting as well. Interestingly enough, in all known examples the positive answer has been confirmed in a quite indirect manner, namely via the Hopf-type rigidity.

\section{Acknowledgements}

We are grateful to Victor Bangert and Eitan Tadmor for useful discussions. We thank also Helmut Hofer, Jürgen Moser and Eduard Zehnder, the 
organizers of the 1995 Dynamical Systems meeting in Oberwolfach, where these results were presented.

\section{References}

[ATF] V. M. Alekseev and V. M. Tikhomirov and S. V. Fomin, Optimal control, Consultants Bureau, New York, 1987.

[Ba] V. Bangert, Minimal foliations and laminations, In Proceedings of ICM 1994 (to appear).

[B] M. Bialy, Convex billiards and a theorem by E. Hopf., Math. Z. 214, 147-154.

[B-P] M. Bialy and L. Polterovich, Hamiltonian systems, Lagrangian tori and Birkhoff's theorem, Math. Ann. 292, 619-627.

[B-I] D. Burago and S. Ivanov, Riemannian tori without conjugate points are flat, GAFA 4, 259-269.

[C] C. Croke, Rigidity and the distance between boundary points, J. Diff. Geometry 33, 445-464.

[C-F] C. Croke and A. Fathi, An inequality between energy and intersection, Bull. London Math. Soc. 22, 489-494.

[C-K] C. Croke and B. Kleiner, On tori without conjugate points, Invent. Math. 120, 241-257.

[D] P. Dazord, Tores Finslériens sans points conjugués, Bull Soc. Math. France 99, 171-192.

[G] L. Green, A theorem of E. Hopf, Michigan Math. J. 3, 31-34.

$[\mathrm{H}]$ E. Hopf, Closed surfaces without conjugate points, Proc. Nat. Acad. Sci. 34, 47-51.

[K] A. Knauf, Closed orbits and converse KAM theory, Nonlinearity 3, 961-973.

School of Mathematich Sciences, Sachler Faculty of Exact Sciences, Tel Aviv University, Tel Aviv 69978 , Israel

E-mail address: bialy@math.tau.ac.il

School of Mathematical Sciences, Sachler Faculty of Exact Sciences, Tel Aviv University, Tel Aviv 69978 , Israel

E-mail address: polterov@math.tau.ac.il 